\title{
Analysis of the Consensus Protocol of Heterogeneous Agents with Time-Delays
}

\author{
Jichao Zhao ${ }^{1}$, Fengzhi Dai ${ }^{1,3}$ *, Yunzhong Song ${ }^{2}$ \\ ${ }^{1}$ College of Electronic Information and Automation, Tianjin University of Science and Technology, \\ China, \\ ${ }^{2}$ School of Electrical Engineering and Automation, Henan Polytechnic University, 454003, China, \\ ${ }^{3}$ Tianjin Tianke Intelligent and Manufacture Technology CO., LTD, China \\ ChinaE-mail:*daifz@tust.edu.cn \\ www.tust.edu.cn
}

\begin{abstract}
In practical engineering applications, the research on the consensus of heterogeneous multi-agents is of great significance. The consensus of multi-agents mainly includes average consensus, maximum consensus, and minimum consensus. The paper studies the average consensus of heterogeneous multiagents, including continuous-time consensus protocol, discrete-time consensus protocol, consensus with time delay, and consensus of switching topology. The knowledge of graph theory is used to describe the system, and the results are simulated and analyzed for the consensus of time delay and switching topology to verify the correctness and effectiveness of the consensus protocol.
\end{abstract}

Keywords: Heterogeneous Multi-Agent; Consensus Protocol; Switching Topology; Time-Delays

\section{Introduction}

A multi-agent system (MAS) is a computing system consisting of multiple robots interacting in an environment. Cooperative robots can be used to perform tasks that are too difficult for a single robot to perform alone. In recent years, as a main branch of distributed system with the development of computer technology and network communication technology, MAS has been widely developed in the fields of distributed sensor network ${ }^{1}$, network congestion control ${ }^{2}$ and flocking movement ${ }^{3}$. The consensus control of MAS is a very important research topic in distributed system.

At present, the research on MAS is still in the initial stage of development on the whole, and there is still a certain distance from the real application. However, its extensive application indicates great development potential, which will definitely attract more experts and scholars to put into the research work in this field and further explore the theory and application of MAS.

MAS has the characteristics of a large number of subjects, a wide distribution of perceptions, and complex communication. There is often information interaction between subjects. Therefore, it is natural to use graph theory to describe this information interaction channel. Consensus protocol is a rule of interaction between agents, which reflects the process of information exchange between adjacent agents.

In this paper, the validity of agent consensus protocol and the relationship between consensus protocol and graph Laplace matrix are verified by analyzing the state of each node in MAS with inertial link. The relationship between algebraic connectivity (Fiedler eigenvalue) of Laplace matrix and convergence rate of MAS is verified by simulation results. 


\section{Preliminaries}

In this paper, the graph theory is used to represent the MAS. A graph $G=(V, E, A)$ is used to represent the topology of the information exchange network between agents. $V=\left\{v_{1}, v_{2}, \ldots, v_{n}\right\}$ indicates that the graph has $n$ nodes and that the system is composed of $n$ multiagents. $E \subseteq\{(i, j): i, j \in V\}$ is the set of edges about $e_{i j}$, indicating that there is information exchange between agent $i$ and $j$, and the information is from $i$ to $j$. $A=$ $\left[a_{i j}\right]$ is the adjacency matrix of the graph, indicating the weight of information exchange between $i$ and $j$. To simplify calculation, let $a_{i j} \in\{0,1\}$. Note that $a_{i j}=a_{j i}$ in the undirected graph, and $a_{i j} \neq a_{j i}$ in the directed graph.

The set of neighbor node $N_{i}=\left\{j \mid j \in V: e_{i j} \in E\right\}$, which represents the set composed of all agents that have information exchange with agent $i$. In the graph, it is represented as the set composed of nodes connected to the wired segment of node $i$. $\operatorname{deg}_{\text {out }}\left(v_{i}\right)$, the out degree of node $i$ is the number of edges starting from node $i$. $\operatorname{deg}_{\text {in }}\left(v_{i}\right)$, the into degree of node $i$ is the number of edges go into node $i$.

The matrix $D \in N_{n \times n}$ is the degree matrix, is defined as follows

$$
\left\{\begin{array}{l}
D_{i i}=\operatorname{deg}_{\text {out }}(i) \\
D_{i j}=0
\end{array}\right.
$$

The graph laplacian matrix is defined as

$$
L=D-A
$$

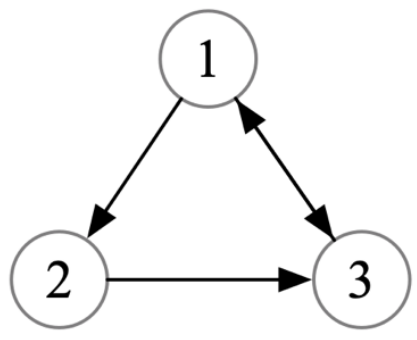

Fig.1. The main global currents

As shown in Fig.1, the system $G$ has three nodes and four edges: $V=\left\{v_{1}, v_{2}, v_{3}\right\}, E=\left\{e_{12}, e_{23}, e_{31}, e_{13}\right\}$, laplacian matrix $L$ equals

$$
L=\left[\begin{array}{lll}
2 & 0 & 0 \\
0 & 1 & 0 \\
0 & 0 & 1
\end{array}\right]-\left[\begin{array}{lll}
0 & 1 & 1 \\
0 & 0 & 1 \\
1 & 0 & 0
\end{array}\right]=\left[\begin{array}{ccc}
2 & -1 & -1 \\
0 & 1 & -1 \\
-1 & 0 & 1
\end{array}\right]
$$

\section{Consensus Protocol}

The individual state of the dynamic MAS is described as follows:

$$
\dot{x}_{i}(t)=-\frac{1}{T_{i}} x_{i}(t)+\frac{K_{i}}{T_{i}} u_{i}(t), \quad i=1,2, \ldots, n .
$$

where $T_{i}, K_{i}$ denote different coefficients of node $i$, and $T_{i} \neq T_{j}, K_{i} \neq K_{j}, \forall i \neq j$;

System to reach consensus, means that the system all individual states are equal, mathematical representation, $x_{i}=x_{j}, \forall i \neq j, i=1,2, \ldots, n$ or $u_{i}=0, i=1,2, \ldots, n$.

When there is a directed spanning tree in communication topology $G$, information interaction between agents can be realized to reach consensus. Therefore, it is assumed that the system topology $G$ contains a directed spanning tree.

In particular, the consensus problem also includes average consensus, maximum consensus and minimum consensus. But it should be noted that the system consensus is equivalent to the average consistency if the graph is undirected or directed symmetric. But if the graph is asymmetric, the consensus of the system is not equivalent to the consensus of the average ${ }^{4}$.

\subsection{Consensus Protocol of Continuous Time}

For continuous time systems (1), the sonsensus protocol is as follows:

$$
u_{i}(t)=\sum_{j \in N_{i}} a_{i j}\left(x_{j}-x_{i}\right)
$$

The agreement can be achieved if and only if the undirected graph is connected or the directed graph exists spanning tree. In the case of topological structure switching, the condition of consensus is that the union of switching topological graphs is undirected graph connected or directed graph exists spanning tree. Specific proof can be found in the literature ${ }^{5}$.

Refer to the topology of Fig.1, (2) can be described as:

$$
\left[\begin{array}{l}
u_{1} \\
u_{2} \\
u_{3}
\end{array}\right]=\left[\begin{array}{c}
-2 x_{1}+x_{2}+x_{3} \\
-x_{2}+x_{3} \\
x_{1}-x_{3}
\end{array}\right]=-\left[\begin{array}{ccc}
2 & -1 & -1 \\
0 & 1 & -1 \\
-1 & 0 & 1
\end{array}\right]\left[\begin{array}{l}
x_{1} \\
x_{2} \\
x_{3}
\end{array}\right]
$$

Combined with the graph laplacian matrix, (2) can be converted into the following form:

$$
u(t)=-L * x(t)
$$




\subsection{Consensus Protocol of Discrete Time}

The discrete-time consensus protocol is as follows:

$$
x_{i}(k+1)=x_{i}(k)+\epsilon u_{i}(k)
$$

$\epsilon>0$ means step size, the length of time for each step.

\subsection{Switching Topology Model}

Since the topological network is not fixed, there is an additional switching signal $k$ is a function of time $t$. The protocol is as follows:

$$
u_{i}(t)=-L_{k} * x(t), \quad k=s(t)
$$

Fiedler eigenvalue is the second smallest eigenvalue of the Laplace matrix of the graph, which is positively correlated with the convergence rate of the graph. If the larger the Fiedler eigenvalue is, the faster the convergence rate of the system will be. On the contrary, the same relationship exists.

\subsection{Time-Delays Model}

The consensus protocol with time delay is:

$$
u_{i}(t)=\sum_{j \in N_{i}} a_{i j}\left(x_{j}(t-\tau)-x_{i}(t-\tau)\right)
$$

Among them, time delay $\tau$ has a threshold value of $\frac{\pi}{2 \lambda_{n}}$.

For a network with the same time delay, assume $G$ is undirected and connected. If and only if at the time delay $\tau$ meet $\tau<\frac{\pi}{2 \lambda_{n}}, \lambda_{n}=\lambda_{\max }(L)$, the system can asymptotically achieve consensus ${ }^{4}$.

\section{Simulink Results}

Given a MAS topology graph, as shown in Fig.2, the system has a total of 6 multi-agents, and the three topologies $G_{a}, G_{b}$ and $G_{c}$ all have the same number of agents, but their topological structures are different.

Let the initial state of the system for each agent is $x_{i}$ as shown in Tab.1. Notice that the average of the initial state of the system is $\operatorname{Ave}\left(X_{0}\right)=5 \neq 0$. Then, the dynamic equation of each node in the MAS is established. The dynamic equation parameters $T_{i}$ and $K_{i}$ are shown in Tab.1, and the parameters obey the random Gaussian distribution. The system state differential equation was established, the system differential equation was solved by software tools, and the system convergence results were drawn as shown in the Fig.3.

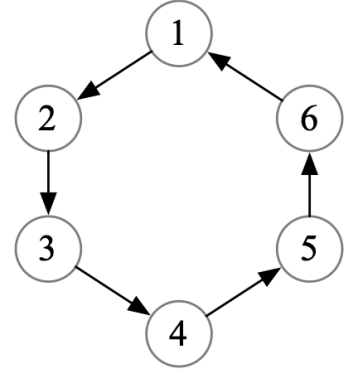

(a)

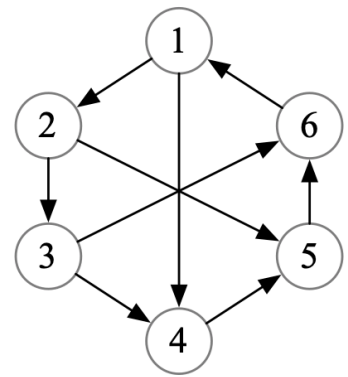

(c)

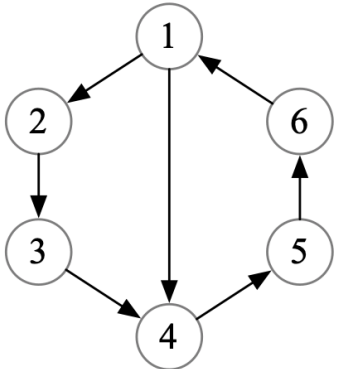

(b)<smiles>CCCCCCC(C)(C)C</smiles>

Fig.2. Topology graph of the system

In Fig.2, the order of switching topology is Ga-Gb-Gc, when $t=0$, the system is topology $\mathrm{A}$, when $t=1$, the system is topology $\mathrm{B}$, and then c, and the loop is carried out in this way.

Tab.1. MAS Parameters

\begin{tabular}{cccc}
\hline$i$ & $x_{i}(0)$ & $T_{i}$ & $K_{i}$ \\
\hline 1 & -20.50 & 0.81 & 0.91 \\
2 & 30.25 & 0.27 & 0.55 \\
3 & -13.25 & 0.95 & 0.49 \\
4 & 24.75 & 0.79 & 0.96 \\
5 & -7.25 & 0.67 & 0.76 \\
6 & 16.00 & 0.70 & 0.03 \\
\hline
\end{tabular}

Fig. 3 shows the input-free convergence graph of the MAS, the fixed topology convergence and the switched topology convergence of the system. When there is no input, the state of the system changes with its inertial link. When input is available, Fiedler eigenvalues of topology $G_{a}, G_{b}$ and $G_{c}$ increase in turn, and the convergence velocity of the three topology graphs in the graph is observed in turn, which can verify the positive 
correlation between Fiedler eigenvalue and the convergence velocity of the system.
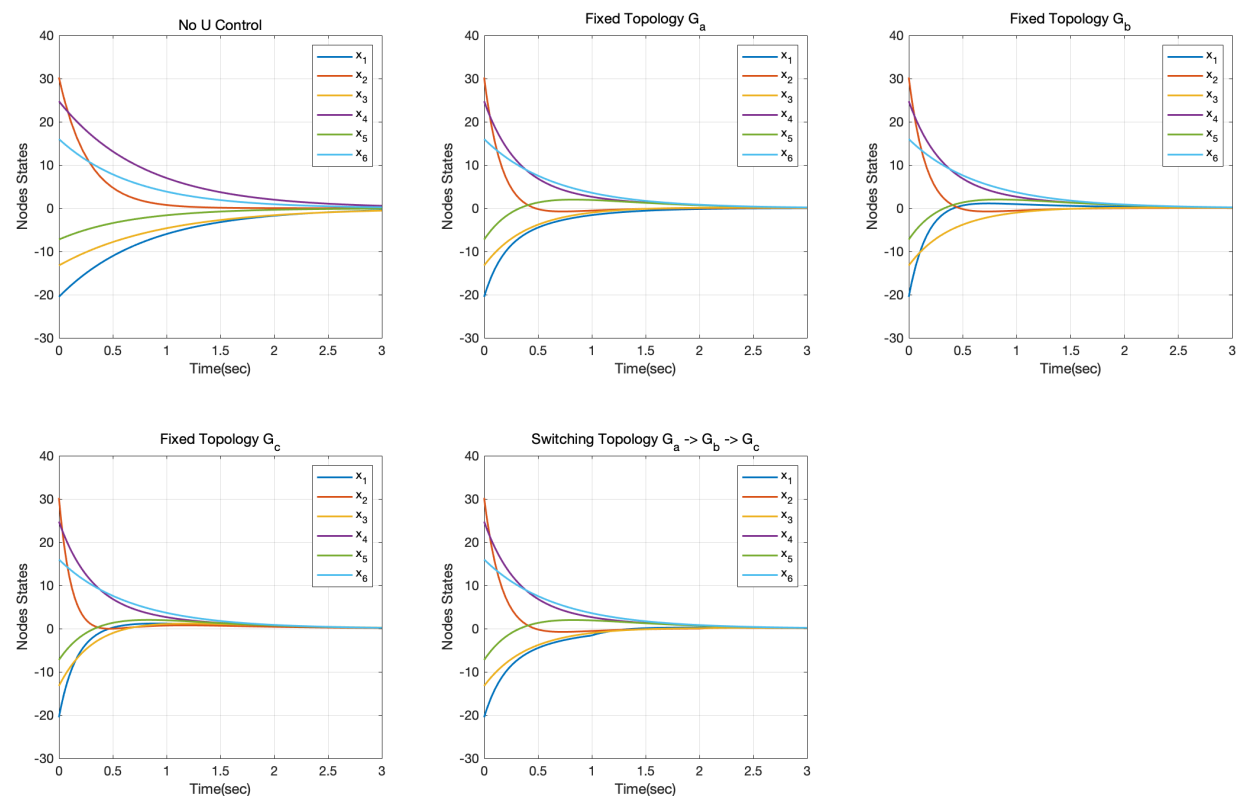

Fig.3. The result of input-free, fixed topology and switched topology

\section{Conclusion}

In this paper, the consensus protocols of MAS are sorted out and each protocol is simply explained. An example is given to illustrate the relationship between multi-agent system and Laplacian matrix in graph theory. The multiagent consensus protocol is simulated, and the protocol is analyzed and verified by combining the knowledge of graph theory and system convergence graph.

\section{References}

1. M. M. I. Khan et al. Efficient and fast convergent consensus algorithms for faulty nodes tracking in distributed wireless sensor networks. 3rd International
Conference on Electrical Engineering and Information Communication Technology, Dhaka, 2016:1-6.

2. Y. Wang, Z. Ma et al. Avoiding Congestion in Cluster Consensus of the Second-Order Nonlinear Multiagent Systems. IEEE Transactions on Neural Networks and Learning Systems, 2018, 29(8): 3490-3498.

3. C. Bhowmick, L. Behera, et al. Flocking control of multiagent system with leader-follower architecture using consensus based estimated flocking center. IECON 2016 42nd Annual Conference of the IEEE Industrial Electronics Society, Florence, 2016:166-171.

4. Olfati-Saber R, Murray R M. Consensus problems in networks of agents with switching topology and timedelays. IEEE Transactions on Automatic Control, 2004, 49(9):1520-1533.

5. Ren W, Beard R W. Distributed consensus in multivehicle cooperative control. London: Springer London, 2008. 